\title{
Helligkeitsschätzungen der Cometen 1886 I (Fabry) und 1886 II (Barnard).
}

Diese beiden Cometen habe ich in den letzten Monaten ihrer Sichtbarkeit am Steinheil'schen Refractor von 6 Zoll Oeffnung bezüglich ihrer Helligkeit wiederholt mit Nachbarsternen verglichen und dabei in der Regel zwei Oculare von sehr verschiedener Stärke (26- und I 12 facher Vergrösserung) benutzt.

Da die centrale Verdichtung in beiden Cometen einen beträchtlichen Durchmesser hatte, und bei Anwendung der starken Vergrösserung ein eigentlicher Kern, wenn ein solcher uberhaupt zu erkennen war, nicht jedesmal mit gleicher Schärfe hervortrat, war mitunter schwer zu entscheiden, wie viel von der hellsten Partie dem Kern zuzurechnen sei, und daher sind diese Schätzungen weniger sicher, als die mit der schwachen Vergrösserung vorgenommenen, bei denen ganz einfach die Helligkeit der gesammten auf einen engen Raum zusammengedrängten Kernscheibe zu ermitteln war. Aus diesem Grunde zeigen auch die bei Anwendung des schwachen Oculars gefundenen Zahlen einen regelmässigeren Gang als die anderen. Helligkeitsspr ünge, deren Betrag die Unsicherheitsgrenzen der Schätzungen in auffallendem Grade übersteigen würde, kamen nicht vor.

Die Vergleichungen wurden, wenn möglich, in der vollen Nacht vorgenommen und nur ausnahmsweise in der Dämmerung, weil ein Comet bei wachsender Tageshelle mehr an Auffälligkeit einbüsst als ein Fixstern. Während der Beobachtungen vor Mitternacht standen die Cometen am Nordwest-, nach Mitternacht am Nordosthimmel. Für die Helligkeiten der Vergleichsterne wurden die Grössenangaben der Durchmusterung angenommen.

Comet 1886 I.

\begin{tabular}{|c|c|c|c|c|c|}
\hline \multirow[b]{2}{*}{ เ 886} & \multirow{2}{*}{$\begin{array}{c}\text { Mittl. } \\
\text { Zeit }\end{array}$} & \multicolumn{2}{|c|}{ Grössenklasse } & \multirow{2}{*}{$\begin{array}{c}\text { Zahl } \\
\text { der } \\
\text { Sterne }\end{array}$} & \multirow[b]{2}{*}{ Bemerkungen } \\
\hline & & $\begin{array}{c}\text { bei } 26 \mathrm{f} \text {. } \\
\text { Vergr. }\end{array}$ & $\begin{array}{l}\text { bei I } 12 \mathrm{f} \\
\text { Vergr. }\end{array}$ & & \\
\hline März ro & $7^{\mathrm{h}}$ & $7^{\mathrm{m}} \cdot 0$ & - & 2 & \\
\hline $3^{\circ}$ & 8 & 6.0 & - & I & \\
\hline April 5 & 15 & 6.0 & $8^{m} \cdot 5$ & 3 & \\
\hline 8 & I $5^{1 / 2}$ & $5 \cdot 5$ & 8.0 & 4 & \\
\hline I I & I $51 / 2$ & 5.0 & 7.0 & 5 & \\
\hline $\begin{array}{l}\text { I } 7 \\
18\end{array}$ & $\begin{array}{l}\text { I } 5 \\
\text { I } 51 / 2\end{array}$ & $\begin{array}{c}5 \cdot 4 \\
4\end{array}$ & 6.4 & $\begin{array}{r}4 \\
--\end{array}$ & $\begin{array}{c}\text { Vollmond } \\
,\end{array}$ \\
\hline
\end{tabular}

Für das freie Auge kam der Comet am i 8. April einem Stern 3. und am 22. April einem Stern 2. Grösse an Auffälligkeit gleich.

Diesen Angaben füge ich noch die Dimensionen von Coma und Schweif an jenen Tagen bei, die solchen Beobachtungen günstig waren.

\begin{tabular}{rr|c|c}
\hline \multicolumn{1}{|c|}{1886} & $\begin{array}{c}\text { Durchm. } \\
\text { der Coma }\end{array}$ & $\begin{array}{c}\text { Länge d. } \\
\text { Schweif. }\end{array}$ \\
\hline Febr. & 3 & $1: 8$ & - \\
April & 5 & 3.5 & $3 / 4^{\circ}$ \\
& 8 & 2.7 & $11 / 2$ \\
11 & 3.2 & $21 / 2$
\end{tabular}

Als Schweiflänge ist jene Dimension angesetzt, bis zu welcher der Schweif mit dem Fernrohr bei 26 facher Vergrösserung mit Bestimmtheit verfolgt werden konnte.

Comet is86 II.

\begin{tabular}{|c|c|c|c|c|c|}
\hline \multirow[b]{2}{*}{188} & \multirow{2}{*}{$\begin{array}{l}\text { Mittl. } \\
\text { Zeit }\end{array}$} & \multicolumn{2}{|c|}{ Grössenclasse } & \multirow{2}{*}{$\begin{array}{c}\text { Zahl } \\
\text { der } \\
\text { Sterne }\end{array}$} & \multirow[b]{2}{*}{ Bemerkingen } \\
\hline & & $\begin{array}{l}\text { bei } 26 \mathrm{f} . \\
\text { Vergr. }\end{array}$ & $\begin{array}{l}\text { bei I I } 2 \mathrm{f} \text {. } \\
\text { Vergr. }\end{array}$ & & \\
\hline \multirow[t]{4}{*}{ März } & $9^{h}$ & $8^{\mathrm{m}} \cdot 5$ & - & 2 & \\
\hline & $81 / 2$ & 8.5 & - & 2 & \\
\hline & $81 / 2$ & 8.0 & - & 2 & \\
\hline & $8^{1 / 2}$ & 7.5 & $8^{\mathrm{m}} \cdot \mathrm{o}$ & 3 & \\
\hline \multirow[t]{10}{*}{ April } & $71 / 2$ & $7 \cdot 5$ & 8.0 & 3 & \\
\hline & 9 & $7 \cdot 3$ & 8.5 & 6 & deutl. Kern \\
\hline & 9 & 7.7 & 9.0 & 4 & $》$ \\
\hline & $81 / 2$ & 7.2 & 8.5 & 2 & \\
\hline & $81 / 2$ & 7.0 & 8.7 & 4 & \\
\hline & 8 & $7 \cdot 5$ & - & I & Mond \\
\hline & $81 / 2$ & 6.7 & 7.0 & 3 & $\gtrsim$ \\
\hline & $83 / 4$ & 6.2 & 7.0 & 3 & \\
\hline & I $5^{1 / 2}$ & $5 \cdot 5$ & 7.0 & 2 & \\
\hline & $83 / 4$ & $5 \cdot 5$ & 7.0 & 2 & \\
\hline Mai & I $4^{1 / 2}$ & 5.0 & 6.0 & - & Kern gut beg \\
\hline
\end{tabular}

Für das freie Auge war der Comet am I 2. Mai so auffällig wie $\beta$ 'Trianguli (3. Gr.).

\begin{tabular}{rr|c|c}
\hline \multicolumn{2}{r|}{ I 886} & $\begin{array}{c}\text { Durchm. } \\
\text { der Coma }\end{array}$ & $\begin{array}{l}\text { Länge d. } \\
\text { Schweif. }\end{array}$ \\
\hline März 30 & $1: 7$ & - \\
April & 7 & 1.5 & - \\
& 22 & 1.7 & - \\
Mai & 7 & 2.7 & $21 / 2^{\circ}$
\end{tabular}

Als die Cometen eine ansehnliche Helligkeit erlangt hatten und die Grössenschätzungen wegen Mangels passender Vergleichsterne unsicher wurden, benutzte ich, um auf einem ganz anderen Weg Anhaltspunkte zur Beurtheilung des Cometenlichtes $\mathrm{zu}$ gewinnen, den Umstand, dass die Gestirne während dieser Periode am Osthimmel vor Sonnen. aufgang zu sehen waren, in der Weise, dass ich den Cometen mit dem Fernrohr bis in die helle Dämmerung verfolgte und den Zeitpunkt notirte, in dem das Licht der Kernscheibe durch das stetig zunehmende Tageslicht verwischt wurde. Dabei beobachtete ich auch die Extinction benachbarter Fixsterne, die somit als Vergleichsobjecte für die Lichtstärke der Cometen dienen können; auch erscheint dadurch der Einfluss leichter 'Trübungen der Atmosphäre wenigstens zum Theil eliminirt. Einige bei stärkeren Trübungen angestellte Beobachtungen lasse ich hier weg. Um eine grössere Zahl von Sternen gleichzeitig mit dem Cometen im Gesichtsfeld zu haben, wurde stets die schwache Vergrösserung benutzt. Die Momente sind in mittlerer Wiener Zeit angegeben. 


\section{Extinctionsbeobachtungen.} Comet I886 I.

April 5. Der Comet verschwand, als die Sterne DM. $+39^{\circ} 5082$, $8^{\mathrm{m}} \cdot 6$, und besonders DM. $+39^{\circ} \cdot 5085,7^{\mathrm{m}} \cdot 7$, noch gut zu sehen waren. Leichtes Federgewölk verhinderte eine genaue Angabe des Zeitpunktes der Extinction.

8. Bei Tagesanbruch verschwanden von den helleren Sternen zuerst DM. $+40^{\circ} .5094,9^{\mathrm{m}} \cdot \mathrm{o}$, und $+40^{\circ} 5099$, 8.9; lange Zeit darauf, nämlich um $16^{\mathrm{h}} 54^{\mathrm{m}}$, der Comet, und $5^{\mathrm{m}}$ später der Stern DM. $+39^{\circ} 5099$, $8^{\mathrm{m}}$. . Luft sehr rein.

11. Als der Stern DM. $+40^{\circ} .5119,8^{\mathrm{m}} \mathrm{r}$, verschwunden war, wurde $3^{\mathrm{m}}$ später, und zwar um $16^{\mathrm{h}} 49^{\mathrm{m}}$, auch der Comet unkenntlich, während DM. $+39^{\circ} 5^{\mathrm{I}} 43$, $7^{\mathrm{m}} \cdot 3$, noch deutlich sichtbar blieb; bald darauf bewölkte sich diese Gegend.

18. Die Kernscheibe des Cometen verschwand nahe gleichzeitig mit DM. $+39^{\circ} 5^{2}, 7^{\mathrm{m}} \cdot 6$, und zwar um $16^{\mathrm{h}} 4 \mathbf{1}^{\mathrm{m}}$; der Stern DM. $+39^{\circ} 5^{6}, 6^{\mathrm{m}} \cdot 6$, war noch $I_{1}^{\mathrm{m}}$ länger $z u$ sehen.

22. Als die beiden Sterne DM. $+36^{\circ} 185,7^{\mathrm{m}} \cdot 8$, und $+36^{\circ} 187,8^{\mathrm{m}} .2$, schon 10 Min. verschwunden waren, konnte um $16^{\mathrm{h}} 33^{\mathrm{m}}$ auch der Comet nicht mehr wahrgenommen werden; gleichzeitig verlor ich die Sterne DM. $35^{\circ} \cdot 18 \mathrm{r}, 7^{\mathrm{m}} \cdot \mathrm{I}$, und $+37^{\circ}{ }^{\mathrm{r}} 90,7^{\mathrm{m}} \cdot \mathrm{o}$, aus dem Auge.

26. Da der Comet von der Sonne nur $14^{\circ}$ im grössten Kreis entfernt war, musste er in der hellen Dämmerung aufgesucht werden. Gleich beim Aufgang stellte ich auf ihn ein, konnte ihn aber erst wahrnehmen, als er $3 \frac{1}{2}^{\circ}$ Höhe erreicht hatte, worauf er bis $16^{\mathrm{h}} 27^{\mathrm{m}}$ sichtbar blieb.

Da der Comet nunmehr die Sonne in AR. überholte, also vom Morgen- nach dem Abendhimmel ruickte, stellte ich am 27. April Abends nach Sonnenuntergang auf ihn ein, aber ohne Erfolg, obschon die Luft rein war; seine Elongation von der Sonne betrug dabei nur noch $12^{\circ}$.

\section{Comet i 886 II.}

Mai I 2. Um $15^{\mathrm{h}} 5^{2^{\mathrm{m}}}$ wurde der Comet nahe gleichzeitig mit dem Stern DM. $+34^{\circ} 3^{6} 3,7^{\mathrm{m}} \cdot 8$, unkenntlich.
Mai I6. Der Comet verschwand um $15^{\mathrm{h}} 39^{\mathrm{m}}$, bald darauf der Stern DM. $+\mathbf{2 9} 9^{\circ} \mathbf{4}^{2} 3,7^{\mathrm{m}} \cdot 5$. Elongation des Cometen von der Sonne gleich $20^{\circ}$.

$\mathrm{Zu}$ den Extinctionsbeobachtungen wäre noch zu bemerken, dass ich die Sterne bei Anwendung eines Refractors mit Uhrwerk voraussichtlich noch etwas länger hätte im Auge behalten können, da sich in diesem Fall die Stelle, auf die man den Blick $2 u$ richten hat, genau markiren lässt. Anders war es im vorliegenden Fall. Hatte man ein Gestirn, wenn es in der vorgtschrittenen Tageshelle bereits an der Grenze der Sichtbarkeit stand, in Folge vorübergehender leichter Trübungen etc. für kurze Zeit verloren, so war es bei abermaliger Klärung in dem nur durch die Hand nachgerückten Fernrohr mitunter nur schwer wieder zu erkennen, weil sich der Blick nicht mehr auf einen bestimmten Punkt des Gesichtsfeldes richten durfte, sondern längs einer Sehne hingleiten musste, zu deren Fixirung übrigens einige am Ocular befindliche Staubtheilcheñ gute Dienste leisteten.

Um ein genähertes Maass für die Helligkeit des Firmamentes zu geben, setze ich noch bei, wie tief die Sonne im Moment der Extinction der Cometen unter dem Horizont stand.

\begin{tabular}{|c|c|c|c|c|c|c|}
\hline \multicolumn{3}{|c|}{ Comet $1886 \mathrm{I}$} & \multicolumn{4}{|c|}{ Comet ${ }_{1} 886 \mathrm{II}$} \\
\hline 1 886 & Mittl. Zt. & $\begin{array}{l}\text { Höhe d. } \\
\text { Sonne }\end{array}$ & I 88 & & Mittl. Zt. & $\begin{array}{l}\text { Höhe d. } \\
\text { Sonne }\end{array}$ \\
\hline $\begin{array}{rr}\text { April } & 8 \\
& \text { I I } \\
18 \\
22 \\
26\end{array}$ & 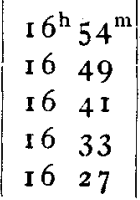 & $\begin{array}{r}-5.5 \\
-5.3 \\
-4.3 \\
-4.4 \\
-4.2\end{array}$ & Mai & $\begin{array}{l}12 \\
16\end{array}$ & $\begin{array}{l}\text { I } 5^{\mathrm{h}} 5^{2^{\mathrm{m}}} \\
\text { I } 539\end{array}$ & $\begin{array}{r}-5.2 \\
-6.2\end{array}$ \\
\hline
\end{tabular}

Für den Cometen 1886 I geht somit aus diesen Zahlen eine Zunahme der Helligkeit hervor, indem der Kern das Tageslicht immer mehr vertrug; für den Cometen 1886 II lässt sich aber aus der geringen Zahl von nur zwei Beobachtungen kein gegru :deter Schluss ziehen.

Wiener Sternwarte 1886 Juni 10.

7. Holetschek.

\section{Observations of Comet 1886 II (Barnard) made at the Leander Mc Cormick Observatory,} with the $66^{\mathrm{em}}$ refractor, power 200.

\begin{tabular}{|c|c|c|c|c|c|c|c|c|c|c|c|c|}
\hline 188 & & Berlin $\mathrm{M} . \mathrm{T}$. & $\Delta \alpha$ & $\Delta \delta$ & Vgl. & $\alpha$ app. & $\log p .4$ & $\delta$ app. & $\log p .4$ & \multicolumn{2}{|c|}{ Red.ad l.app. } & $*$ \\
\hline Jan. & 19 & $15^{\mathrm{h}} 34^{\mathrm{m}} 5^{\mathrm{I}^{\mathrm{s}}}$ & $+o^{m} 42.89$ & 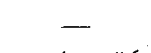 & 9 & - & 9.508 & - & - & - & - & I \\
\hline & I9 & 1537 & $=$ & $-I^{\prime} 42.6$ & 3 & - & - & - & 0.632 & -- & - & I \\
\hline Febr. & 13 & I 44450 & +018.37 & - & I 8 & - & 9.632 & - & - & - & - & 2 \\
\hline & 13 & 1410 & $-\quad-$ & $\begin{array}{ll}-1 & 3 \cdot 3\end{array}$ & 6 & - & - & - & 0.596 & - & $\ldots$ & 2 \\
\hline & 17 & I3 $27 \quad 54$ & +133.66 & 一 & I 2 & - & 9.557 & - & - & -0.16 & - & 3 \\
\hline & I 7 & I $4 \quad 53$ & 一 & +324.6 & 5 & - & - & 一 & 0.597 & - & $-4 " 1$ & 3 \\
\hline & I 7 & $1327 \quad 54$ & -03.18 & - & 12 & - & 9.557 & - & - & - & - & 4 \\
\hline
\end{tabular}

